\title{
Relic Neutralino Densities and Detection Rates with Nonuniversal Gaugino Masses
}

\author{
Andreas Birkedal-Hansen \\ Department of Physics, University of California, Berkeley, CA 94720, USA and \\ Theoretical Physics Group, Lawrence Berkeley National Laboratory, Berkeley, CA 94720, USA \\ Brent D. Nelson \\ Michigan Center for Theoretical Physics, University of Michigan, Ann Arbor, MI 48109, USA
}

(Dated: October 25, 2018)

\begin{abstract}
We extend previous analyses on the interplay between nonuniversalities in the gaugino mass sector and the thermal relic densities of LSP neutralinos, in particular to the case of moderate to large $\tan \beta$. We introduce a set of parameters that generalizes the standard unified scenario to cover the complete allowed parameter space in the gaugino mass sector. We discuss the physical significance of the cosmologically preferred degree of degeneracy between charginos and the LSP and study the effect this degree of degeneracy has on the prospects for direct detection of relic neutralinos in the next round of dark matter detection experiments. Lastly, we compare the fine tuning required to achieve a satisfactory relic density with the case of universal gaugino masses, as in minimal supergravity, and find it to be of a similar magnitude. The sensitivity of quantifiable measures of fine-tuning on such factors as the gluino mass and top and bottom masses is also examined.
\end{abstract}

PACS numbers: 12.60.Jv,04.65.+e,14.80.Ly,95.35.+d

\section{Introduction}

The perplexing question of the nature of the missing non-baryonic matter in the universe continues to be an active area of research. In supersymmetry, the lightest supersymmetric particle (LSP) tends to be a neutral gaugino, which is stable if the assumption of R-parity conservation is imposed, and its weak-scale self-interaction rate in the early universe make it an attractive candidate to fill this role. Not surprisingly, then, relic neutralinos continue to be the favored candidate for the missing matter density of the universe.

Most phenomenological studies of the parameter space in supersymmetric models that give rise to the cosmologically preferred thermal relic density [1], 2] of $0.1 \leq$ $\Omega_{\chi} \mathrm{h}^{2} \leq 0.3$ focus on the minimal supergravity model (mSUGRA) with its unified gaugino mass $m_{1 / 2}$ and unified scalar mass $m_{0}$. In such constrained models of the $\operatorname{MSSM}^{1}$ a typical point in the $\left(m_{1 / 2}, m_{0}\right)$ plane that would otherwise be quite "natural" from the point-ofview of solving the hierarchy problem of the electroweak sector tends to produce far too much relic neutralino density in the early universe to be compatible with the known age of the universe. A sizable portion of the parameter space that does not come into conflict with the bound $\Omega_{\chi} \mathrm{h}^{2} \lesssim 0.3$ is instead in conflict with the bound on the lightest CP-even Higgs mass coming from LEP, particularly for low values of the parameter $\tan \beta$.

This disturbing situation was pointed out in our pre-

\footnotetext{
1 The class of models which we refer to as mSUGRA is also commonly known as the constrained MSSM, or "CMSSM," in the literature.
}

vious study [3], where it was observed that significant improvement can be achieved if the assumption of universal gaugino masses is relaxed. The problematic behavior of the mSUGRA model is a result of the overwhelming B-ino content of the LSP and the large mass difference between the LSP and the next lightest supersymmetric particle (NLSP) which holds over most of the parameter space in such models. Combined with the need for a large universal gaugino mass to accommodate a Higgs mass of $115 \mathrm{GeV}$ these properties lead to an excess of relic B-inos outside of certain special areas where resonant annihilation or coannihilation processes dominate. When this assumption of gaugino mass universality is not valid - in particular when the $\mathrm{W}$-ino mass $M_{2}$ is less than the B-ino mass $M_{1}$ at the initial supersymmetry breaking scale $\Lambda_{\mathrm{UV}}$ - the increased $\mathrm{W}$-ino content of the LSP and reduced mass difference $\Delta m \equiv m_{\chi^{ \pm}}-m_{\chi_{1}^{0}}$ between the LSP neutralino $\chi_{1}^{0}$ and NLSP chargino $\chi^{ \pm}$can have dramatic effects on the depletion of relic neutralinos in the early universe. This will open up new areas of MSSM parameter space with acceptable relic densities. Quite apart from this improvement in the dark matter arena, such nonuniversalities in the gaugino sector are a common feature of string-derived effective theories. It was further suggested recently [4] that nonuniversalities in the gaugino sector may also play an important role in ameliorating the apparent fine-tuning of the MSSM required in the electroweak sector as well.

In the current work we utilize newly-available calculation tools to extend the analysis to include the important large- $\tan \beta$ region which has been of great interest recently in the context of minimal supergravity models. We pay particular attention to the behavior of that area of parameter space in which resonant annihilation through heavy Higgs states occurs when gaugino mass nonuniver- 
sality is introduced. We find that the viable parameter space is enlarged, with significant regions allowing for heavy scalars and relatively light gauginos. The physical significance behind this new parameter region is investigated and the sensitivity of these results to the value of the gluino mass is explored. This analysis forms the bulk of Section 1 .

Just as changing the relative size of the gaugino mass parameters can have large effects on the number and nature of coannihilation channels available to the relic LSP in the early universe, so too can it have profound impacts on the interaction rates of surviving relics with current dark matter detectors. In Section [I] we investigate the prospects for direction detection of relic neutralinos in our local halo in the next round of future detectors. While nonuniversalities that give rise to a relic density in the preferred range typically generate interaction crosssections too small for current detectors such as DAMA or CDMS, we find a significant region of the preferred parameter space will be probed by the next generation of Germanium and Xenon-based detectors.
Lastly, in an effort to quantify the difference between the mSUGRA paradigm and the case with nonuniversal gaugino masses, we adopt a fine-tuning sensitivity parameter similar to that of Ellis and Olive [5]. We calculate the sensitivity of the neutralino relic density to small variations in the high scale parameters, including input values such as the top and bottom quark masses. In Section III we compare the typical amounts of tuning in universal and nonuniversal scenarios, and comment upon the implications, before concluding.

\section{RELIC DENSITY OF LSP NEUTRALINOS}

We are interested in studying the present density of neutralinos assuming they were once in thermal equilibrium with radiation in the early universe. The lightest such neutralino will be the LSP in all models we will consider. The mass and couplings of the neutralino sector at tree level are determined by the mass matrix

$$
\left(\begin{array}{cc}
M_{1} & 0 \\
0 & M_{2} \\
-\sin \theta_{W} \cos \beta M_{Z} & \cos \theta_{W} \cos \beta M_{Z} \\
\sin \theta_{W} \sin \beta M_{Z} & -\cos \theta_{W} \sin \beta M_{Z}
\end{array}\right.
$$

which is written in the $\left(\widetilde{B}, \widetilde{W}, \widetilde{H}_{d}^{0}, \widetilde{H}_{u}^{0}\right)$ basis, where $\widetilde{B}$ represents the B-ino, $\widetilde{W}$ represents the neutral W-ino and $\widetilde{H}_{d}^{0}$ and $\widetilde{H}_{u}^{0}$ are the down-type and up-type Higgsinos, respectively. The parameters $M_{1}$ and $M_{2}$ in (11) are the soft supersymmetry-breaking gaugino masses for the B-ino and W-ino, respectively while $\mu$ is the supersymmetric Higgsino mass parameter. The ratio of Higgs vevs is given by the parameter $\tan \beta=v_{u} / v_{d}$ with $v_{u}$ the vacuum expectation value of the up-type Higgs and $v_{d}$ the vacuum expectation value of the down-type Higgs. We will assume that all of the parameters in (11) are real for this analysis. ${ }^{2}$

The content of the lightest neutralino can be parameterized by writing the LSP as

$$
\chi_{1}^{0}=N_{11} \widetilde{B}+N_{12} \widetilde{W}+N_{13} \widetilde{H}_{d}^{0}+N_{14} \widetilde{H}_{u}^{0}
$$

with the normalization $N_{11}^{2}+N_{12}^{2}+N_{13}^{2}+N_{14}^{2}=1$. When the parameter $\mu$ is much larger than the Z-boson mass it is clear that the proportion of $\mathrm{W}$-ino content to Bino content in the LSP is determined by the ratio of their soft masses, which we denote by $r \equiv M_{2} / M_{1}$. In

\footnotetext{
2 See, however, Ref. 6 for the implications on neutralino relic densities when this assumption is relaxed.
}

$$
\left.\begin{array}{cc}
-\sin \theta_{W} \cos \beta M_{Z} & \sin \theta_{W} \sin \beta M_{Z} \\
\cos \theta_{W} \cos \beta M_{Z} & -\cos \theta_{W} \sin \beta M_{Z} \\
0 & -\mu \\
-\mu & 0
\end{array}\right),
$$

mSUGRA this parameter is defined to be $r=1$ at some high-energy input scale, usually taken to be the GUT scale $\Lambda_{\mathrm{UV}}=\Lambda_{\mathrm{GUT}}=2 \times 10^{16} \mathrm{GeV}$. Upon renormalization group (RG) evolution of these parameters to the electroweak scale this implies $M_{1} \simeq \frac{1}{2} M_{2}$ and the lightest eigenvalue of (11) is thus overwhelmingly B-ino in content $\left(N_{11} \simeq 1\right)$. In a more general scenario the neutralino sector would be defined by four parameters at the electroweak scale: $\left\{\tan \beta, \mu, r, M_{1}\right\}$.

In practice the magnitude of the $\mu$ parameter is determined at the electroweak scale by enforcing proper electroweak symmetry breaking (EWSB). Here this will be performed by computing the complete one loop corrected effective potential $V_{1-\text { loop }}=V_{\text {tree }}+\Delta V_{\text {rad }}$. The correction $\Delta V_{\text {rad }}$ includes radiative effects from the entire superpartner spectrum and thus involves all of the parameters of the soft supersymmetry-breaking Lagrangian. The effective $\mu$-term $\bar{\mu}$ is calculated from the EWSB condition

$$
\bar{\mu}^{2}=\frac{\left(m_{H_{d}}^{2}+\delta m_{H_{d}}^{2}\right)-\left(m_{H_{u}}^{2}+\delta m_{H_{u}}^{2}\right) \tan \beta}{\tan ^{2} \beta-1}-\frac{1}{2} M_{Z}^{2},
$$

where $\delta m_{H_{u}}$ and $\delta m_{H_{d}}$ are the second derivatives of the radiative corrections $\Delta V_{\text {rad }}$ with respect to the up-type and down-type Higgs scalar fields, respectively. The sign of the $\mu$ parameter is not determined and left as a free 

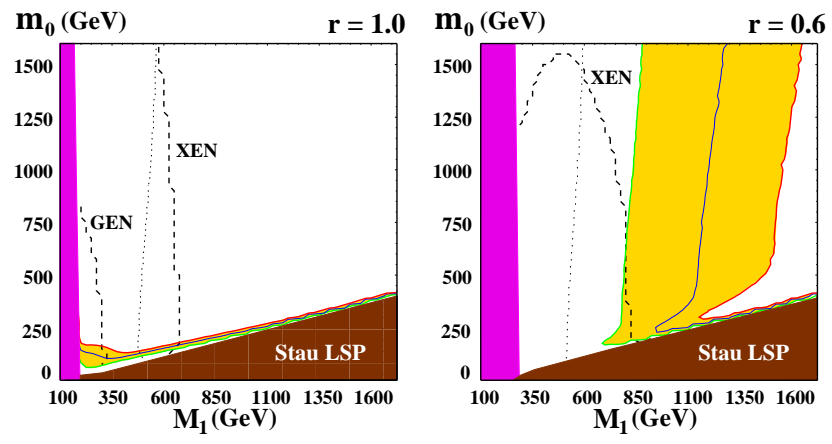

FIG. 1: Neutralino Relic Densities for $\tan \beta=5$. The preferred neutralino relic density regions (light shading) are displayed for $r=1$ (left panel) and for $r=0.6$ (right panel). Dark shaded regions in the lower right are excluded due to a charged LSP. Medium shaded regions on the left are excluded by LEP bounds on the chargino mass. The Higgs mass contour of $m_{h}=113 \mathrm{GeV}$ is given by the dotted vertical line. The dashed contours are a rough estimate of the direct detection reach of the GENIUS ("GEN") and XENON ("XEN") experiments.

\section{parameter.}

Determination of $\mu$ through (3), as well as the determination of the one loop radiative corrections to the neutralino mass matrix (11), involve the remainder of the supersymmetry breaking soft terms. These soft terms will also be necessary to translate low energy values in (11) into high energy input values by solving the renormalization group equations (RGEs). We wish to isolate the effects of gaugino mass nonuniversality so we choose to consider a universal scalar mass $m_{0}$ and universal trilinear A-term $A_{0}$ as in minimal supergravity. We are thus led to consider the seven-dimensional parameter space we refer to as $r S U G R A$ given by

$$
\left\{\tan \beta, \operatorname{sgn}(\mu), r, M_{1}, M_{3}, m_{0}, A_{0}\right\} .
$$

The effect of the trilinear coupling $A_{0}$ is negligible for the physics we are considering here so we will set $A_{0}=0$ throughout. We will also restrict our attention to positive $\mu$. This is very similar to the parameter space originally studied by Mizuta, Ng and Yamaguchi [7], where a trio of specific values for the parameter $r$ were investigated. The effect of gaugino mass nonuniversality on neutralino dark matter in certain specific models has also been computed recently in Ref. 8 and 9. The latter appeared as we completed this analysis and we will comment on areas of agreement with that work during the following sections.

Translation of the parameter set (4) from their values at the high scale (we will assume $\Lambda_{\mathrm{UV}}=\Lambda_{\mathrm{GUT}}$ ) is performed using the Fortran code SuSpect [10] which solves the RGEs at two loops between the scale $\Lambda_{\text {GUT }}$ and $M_{Z}$ through an iterative procedure that takes into account proper thresholding of superpartners. Proper EWSB is imposed and the one loop corrected $\mu$ parameter is determined. The complete superpartner spectrum is calculated including one loop radiative corrections.
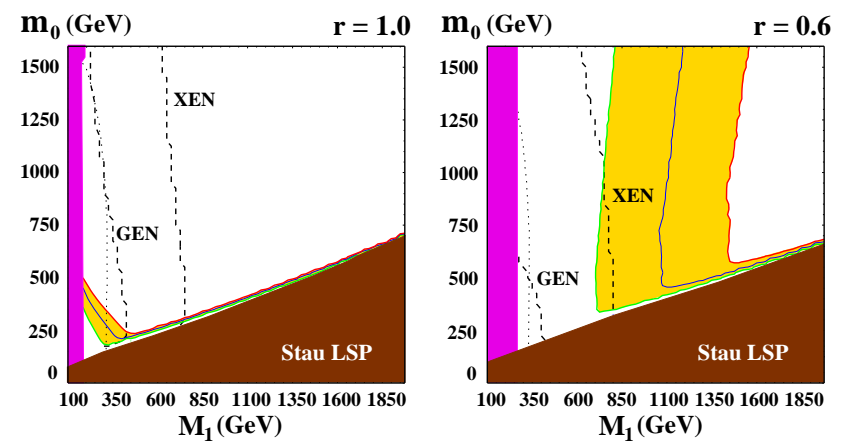

FIG. 2: Neutralino Relic Densities for $\tan \beta=35$. The preferred neutralino relic density regions (light shading) are displayed for $r=1$ (left panel) and for $r=0.6$ (right panel). Dark shaded regions in the lower right are excluded due to a charged LSP. Medium shaded regions on the left are excluded by LEP bounds on the chargino mass. The Higgs mass contour of $m_{h}=113 \mathrm{GeV}$ is given by the dotted vertical line. The dashed contours are a rough estimate of the direct detection reach of the GENIUS ("GEN") and XENON ("XEN") experiments.

The soft supersymmetry breaking parameters at the weak scale are then passed to the $\mathrm{C}$ code micrOMEGAs 11. to perform the relic density calculation. This consists of computing a thermally averaged annihilation cross section times LSP velocity $\langle\sigma v\rangle_{\text {ann }}$ and freeze-out temperature $x_{F}=T_{F} / m_{\chi_{1}^{0}}$ for the LSP $\chi_{1}^{0} 112,13,14,15,16$. Freeze-out occurs when the relic density of the LSP no longer tracks the density of relativistic degrees of freedom in the universe but is instead nearly constant. The freezeout temperature is defined in micrOMEGAs as the temperature at which the relic density of $\chi_{1}^{0}$ becomes roughly twice the value that would obtain in equilibrium. The power of micrOMEGAs lies in its inclusion not just of direct annihilation processes involving two neutralinos such as $\chi_{1}^{0} \chi_{1}^{0} \rightarrow \ell^{+} \ell^{-}$, but also of all possible coannihilation channels involving the relic LSP such as $\chi_{1}^{0} \chi^{ \pm} \rightarrow W^{ \pm} Z$ and $\chi_{1}^{0} \tilde{\tau} \rightarrow \gamma \tau$. These processes are known to be crucial to a proper calculation of the relic density whenever the masses of the LSP and of the coannihilating particle are nearly degenerate $[17,18,19,20,21,22,23,24$.

\section{A. The case of gluino/b-ino equality}

As mentioned in the introduction, the typical mSUGRA analysis has the luxury of displaying results in the $\left\{m_{1 / 2}, m_{0}\right\}$ plane for a given value of $\tan \beta$. Our parameter set given in (4) introduces two new degrees of freedom for the gaugino sector, so we will begin our analysis by exhibiting the neutralino relic density in the $\left(M_{1}, m_{0}\right)$ plane for specific values of $r$ and $\tan \beta$. In order to make contact with the familiar results of recent mSUGRA studies [25, 26, 27, 28, 29] we will set $M_{3}=M_{1}$ at the initial GUT scale in this subsection. Since the bulk 

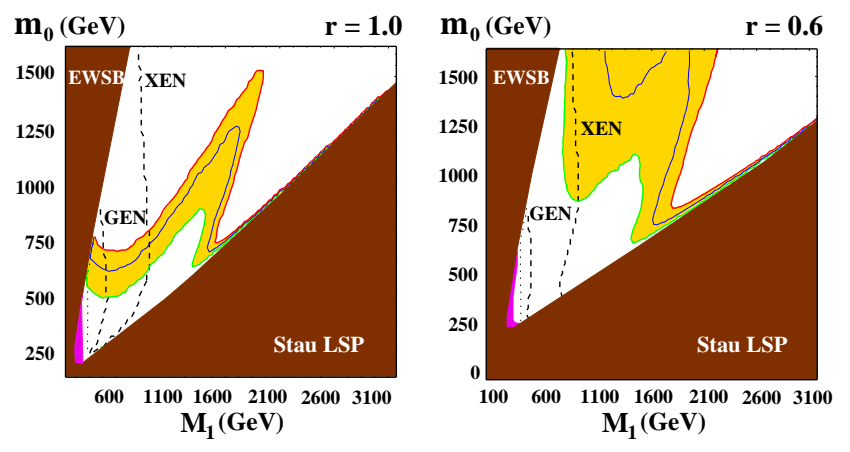

FIG. 3: Neutralino Relic Densities for $\tan \beta=50$. The preferred neutralino relic density regions (light shading) are displayed for $r=1$ (left panel) and for $r=0.6$ (right panel). Dark shaded regions in the lower right are excluded due to a charged LSP, while those in the upper left are excluded due to improper EWSB. Medium shaded regions in the lower left are excluded by LEP bounds on the chargino mass. The Higgs mass contour of $m_{h}=113 \mathrm{GeV}$ is given by the dotted vertical line. The dashed contours are a rough estimate of the direct detection reach of the GENIUS ("GEN") and XENON ("XEN") experiments.

of the RGE effects of a given value of $m_{1 / 2}$ in unified models is related to the value of $M_{3}$, this choice most closely mimics the RGE behavior of the universal mSUGRA scenario.

The results of this analysis are shown in Figures 1 , 目 and 3 for values of $\tan \beta$ of 5,35 and 50 respectively. In each of these figures, we display available parameter space in the $\left(M_{1}, m_{0}\right)$ plane, with $M_{1}$ and $m_{0}$ being the soft supersymmetry breaking parameters at the initial (GUT) scale, for $r=1$ and $r=0.6$. The former value recovers the case of minimal supergravity, while the latter value was observed in Ref. 3 to be the area where nonuniversality in the gaugino mass sector reaches a critical value for which the preferred relic density becomes roughly independent of scalar mass values. The lightly shaded area in each region is the area of the parameter space preferred by cosmology, with $0.1 \leq \Omega_{\chi} \mathrm{h}^{2} \leq 0.3$. The left-most contour (green) contour bordering this region is the contour of $\Omega_{\chi} \mathrm{h}^{2}=0.1$ while the rightmost (red) contour bordering the region is the contour of $\Omega_{\chi} \mathrm{h}^{2}=0.3$. Recent analysis of all applicable cosmological data 30 suggests a narrower region for lower values of $\Omega_{\chi} \mathrm{h}^{2}$ may be preferred by observational data. We therefore provide the (blue) contour for $\Omega_{\chi} \mathrm{h}^{2}=0.2$, within the preferred region, as a guide to this more restrictive parameter space.

The darkly shaded areas in each plot are ruled out by requiring the lightest neutralino to be the lightest superpartner (the region in the lower right) or by requiring that correct electroweak symmetry breaking occurs (the region in the upper left on some plots). While it has become fashionable for dark matter calculational tools to include computations of indirect constraints on supersymmetry such as $\operatorname{BR}(b \rightarrow s \gamma)$ and $\left.\delta\left(a_{\mu}\right)\right|_{\text {susy }}$, we have chosen not to include such information in our figures to reduce clutter. ${ }^{3}$ We have checked, however, that with our choice of $\mu>0$ the conservative 1- $\sigma$ lower limit on the branching ratio for inclusive decays $\mathrm{BR}(b \rightarrow s \gamma) \geq 2.2 \times 10^{-4}$ coming from CLEO [31] is satisfied for all of the parameter choices we exhibit. So too is the conservative upper bound [32] on the SUSY contribution to the muon anomalous magnetic moment $\left.\delta\left(a_{\mu}\right)\right|_{\text {SuSY }} \leq 90 \times 10^{-10}$ [33] for all but the slimmest region in the lower left corner of these figures - a region irrelevant to the parameter region we are exploring in this paper. Two key experimental constraints which we do display are the Higgs and chargino mass limits from LEP. Regions excluded by the LEP limit on the mass of the lightest chargino $m_{\chi_{1}^{ \pm}} \geq 103 \mathrm{GeV}$ 34, 35] are indicated by the medium (magenta) shaded regions on the left of the plots. We have chosen to be conservative and indicate the contour of $m_{h}=113 \mathrm{GeV}$ for the lightest CP-even Higgs mass by the dotted line as an indication of the area excluded by LEP [36].

Finally, the estimated detection reach of the proposed Germanium detector GENIUS, and the next-generation liquid Xenon-based XENON detector, are indicated by the heavy dashed lines labeled "GEN" and "XEN," respectively. These are obtained by taking the estimated minimal cross section for neutralino-nucleon elastic scattering, $\sigma_{\chi p}$, capable of producing a signal in GENIUS [37] and XENON [38], as a function of the LSP mass $m_{\chi_{1}^{0}}$. This is translated into a contour in the $\left(M_{1}, m_{0}\right)$ plane and normalized for a neutralino relic density of $\Omega_{\chi} \mathrm{h}^{2}=$ 0.1. We will discuss these contours in more detail in Section 1 .

We begin with the cases for low to moderate $\tan \beta$ in Figures 1 and 2, which display similar features. For $r=1$ there is very little parameter space available for which the Higgs exclusion bound is not violated, particularly for low $\tan \beta$. One is required to be in the thin coannihilation tail where the lightest stau is almost exactly degenerate with the lightest neutralino. This situation is well-known in mSUGRA (the $r \rightarrow 1$ limit of rSUGRA). However, once $r$ is lowered towards the critical value of $r=0.6$ we see that a wide plume opens up, independent of $m_{0}$, that is perfectly consistent with all experimental constraints arising from accelerators. For both low and moderate $\tan \beta$ this preferred region for $r=0.6$ arises at relatively large values of $M_{1}$, implying relatively large values of the gluino mass, and thus occurs in areas of the parameter space where the Higgs mass can easily exceed the LEP limit.

For extremely large values of $\tan \beta$, as Figure 3 demonstrates, the Higgs mass constraint is satisfied throughout most of the parameter space. Here resonant annihilation of neutralinos can proceed through an s-channel exchange of neutral heavy Higgs states, particularly the

\footnotetext{
${ }^{3}$ It should be noted that micrOMEGAs also computes such quantities, as well as applying direct collider limits.
} 
pseudoscalar. This generates an increase in the cosmologically preferred region, even for $r=1$. The large size of this region is a direct result of the large width of the CP-odd Higgs mass in this high $\tan \beta$ regime and has generated much recent interest [21, 29, 39, 40, 41, 42. The position, and indeed even the existence, of this large region for any given value of $\tan \beta$ is very sensitive to the Yukawa couplings of the bottom and top quarks 41. The pole masses we used in Suspect for this analysis were $m_{t}^{\text {pole }}=175.0 \mathrm{GeV}$ and $m_{b}^{\text {pole }}=4.9 \mathrm{GeV}$. This choice is slightly higher than the default setting of $m_{b}^{\text {pole }}=4.6 \mathrm{GeV}$ in Suspect, but we find this value allows us to match our results most closely with those of Refs. 39, 40, 41 for $\tan \beta=50$. This should be seen as evidence of the sensitivity of high $\tan \beta$ results to the subtleties of one's RGE code and how one treats the NLO corrections to the Higgs sector. ${ }^{4}$ From the right panel of Figure 3 it is clear that the presence of this Higgs pole region continues to distort the allowed parameter space as we increase the degree of nonuniversality in the B-ino/W-ino system, but the sensitivity of this allowed space to Yukawa couplings is greatly reduced. We will return to this issue in Section III below.

It would be tempting to conclude from Figures 1 through 3 that relaxing the unification constraint on the gaugino masses will increase the supersymmetric parameter space that can properly account for the cold dark matter of the universe. And while it is certainly true that any particular point in the $\left(M_{1}, m_{0}\right)$ plane - particularly those at larger values of these soft parameters - can now always accommodate the cosmologically preferred relic density for some particular choices of $r$ and $\tan \beta$, the size of the allowed areas in these figures is somewhat deceptive. More information can be obtained by exchanging the variable $m_{0}$ for $r$, since it is this parameter that is more directly tied to the physics that determines the eventual LSP relic density.

For this reason we plot the neutralino relic density in the $\left(M_{1}, r\right)$ plane in Figure 4 for $\tan \beta=5$ (the top pair of plots), $\tan \beta=35$ (the middle pair of plots) and for $\tan \beta=50$ (the bottom pair of plots). In this case we have fixed the scalar mass to two different values to isolate the effects of the gaugino sector parameters alone. Displayed in this manner it is clear that the allowed region in gaugino mass space is a narrow band that extends from the mSUGRA limit of $r=1$ in the top left, where the region of direct annihilation between very light LSPs is ruled out by the LEP chargino mass limits, eventually extending to lower values of $r$, and then connecting continuously with the stau coannihilation region for low values of $m_{0}$. We believe that this space is the natural one for discussing neutralino dark matter in nonuniversal gaugino mass models: it conveys far more information

\footnotetext{
${ }^{4}$ For an analysis of mSUGRA parameter space involving the default SuSpect settings, one may consult Ref. 28.
}
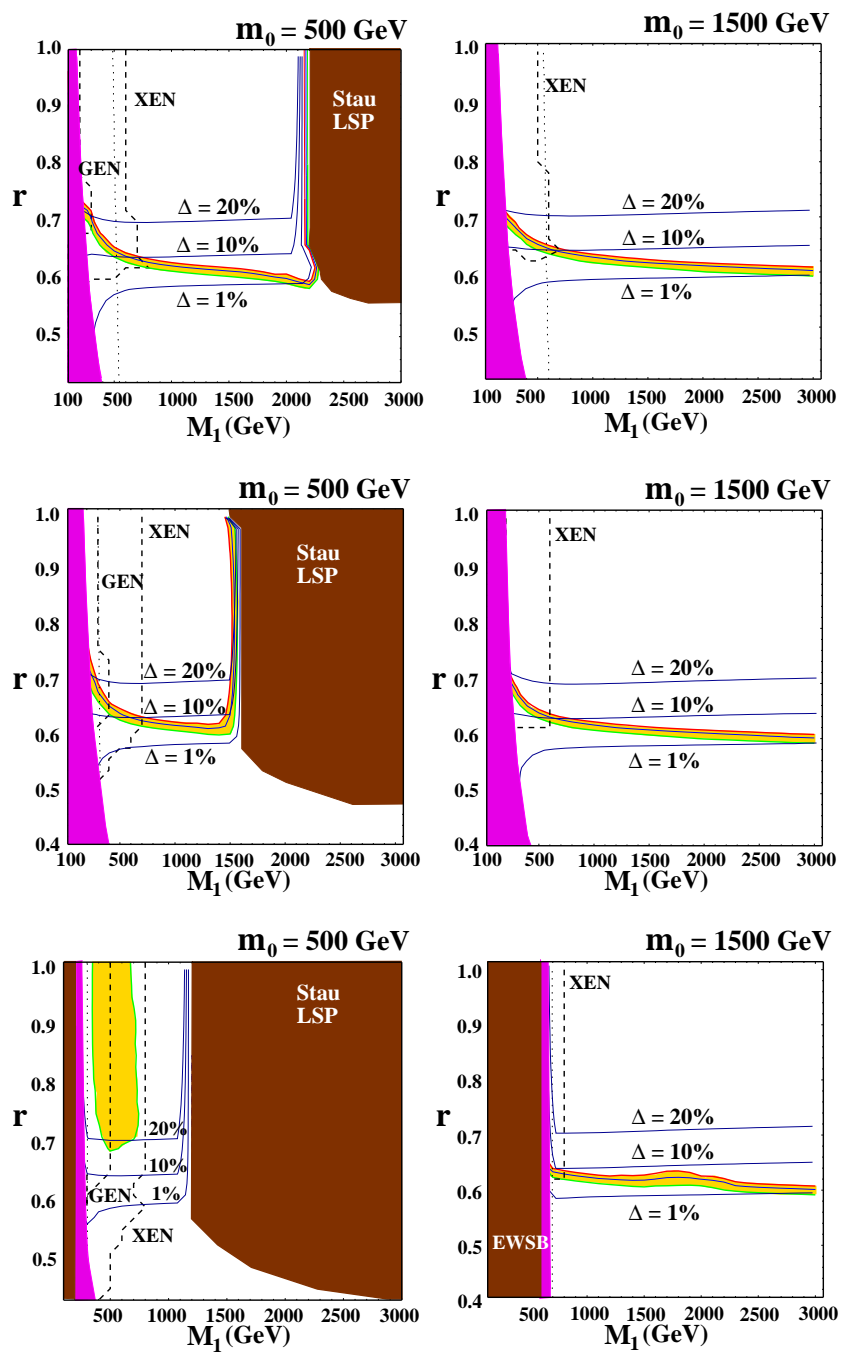

FIG. 4: Preferred Region in Gaugino Mass Space. Top panels are for $\tan \beta=5$, middle panels are for $\tan \beta=35$ and bottom panels are for $\tan \beta=50$. The preferred neutralino relic density regions (light shading) are displayed for universal scalar mass $m_{0}=500 \mathrm{GeV}$ (left panels) and for $m_{0}=1500 \mathrm{GeV}$ (right panels). The dark shaded region in the upper right of the left panels is excluded due to the charged LSP, while the region on the left in the $\tan \beta=50$ case is excluded by improper EWSB. The medium shaded regions on the left are excluded by LEP bounds on the chargino mass. The Higgs mass contour of $m_{h}=113 \mathrm{GeV}$ is given by the dotted vertical line. Contours of constant degeneracy parameter $\Delta$ are given by the thin near-horizontal lines. We have indicated by the dashed contours a rough estimate of the direct detection reach of GENIUS ("GEN") and XENON ("XEN"), where applicable.

on neutralino dark matter than the typical $\left(M_{1 / 2}, m_{0}\right)$ plane in that the correlation between degeneracy and relic density is readily apparent.

To elaborate, we have plotted contours of constant de- 


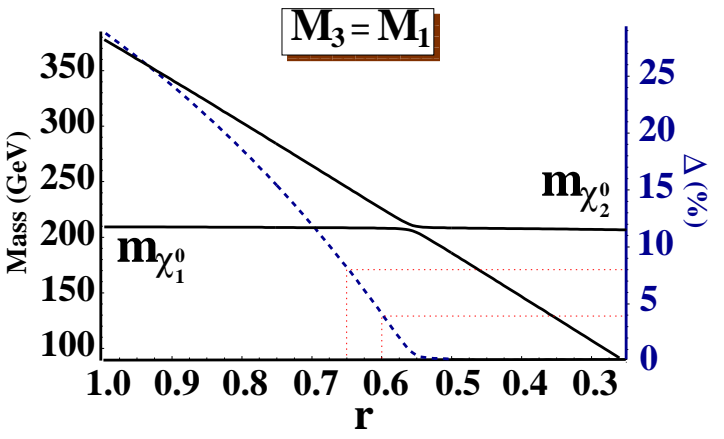

FIG. 5: Preferred Mass Degeneracy in the Gaugino System when $M_{3}=M_{1}$ and $\tan \beta=35$. The left vertical axis measures the mass (solid lines) of the lightest and nextto-lightest neutralinos $\chi_{1}^{0}$ and $\chi_{2}^{0}$, respectively, as a function of the parameter $r$. The right vertical axis measures the degree of degeneracy (dashed line) between the LSP and the chargino as given by the parameter $\Delta$, in percent, as a function of the same parameter. The cosmologically interesting region $0.65 \gtrsim r \gtrsim 0.60$ is indicated by the red dotted lines.

generacy degree $\Delta$ defined by

$$
\Delta \equiv \frac{m_{\chi_{1}^{ \pm}}-m_{\chi_{1}^{0}}}{\bar{m}}
$$

where $\bar{m}$ is the average of the chargino and LSP masses. For moderate to large values of the LSP mass, as determined by the parameter $M_{1}$ at the GUT scale, the preferred region of parameter space is in the neighborhood of $r \simeq 0.6$ where the degree of degeneracy between the two mass eigenstates is roughly $\Delta=5 \%$. In this region of the parameter space the degree of degeneracy is just sufficient to allow coannihilation processes between the chargino and LSP to sufficiently reduce the otherwise large thermal relic density of the lightest neutralino [3]. Clearly, as this degeneracy is increased still further at lower values of the parameter $r$ the coannihilation processes will be too efficient and the lightest neutralino ceases to be an attractive dark matter candidate without some method of non-thermal production. This is, for example, the case in models of anomaly-mediation for which $r_{\mathrm{AMSB}}=M_{2} / M_{1}=5 / 33 \simeq 0.15$ at the GUT scale [43].

The importance of this special value of $\Delta$, and hence of the phenomenological parameter $r=M_{2} / M_{1}$, is no great mystery. As was pointed out by Griest \& Seckel [17] the coannihilating particle must be thermally accessible to the LSP near the time of freeze-out for coannihilation processes to have a significant impact on the overall relic density. Typical freeze-out temperatures in the parameter space we consider are on the order of $T_{F} \simeq m_{\chi_{1}^{0}} / 20$, implying a degeneracy on the order of a few percent is required to make coannihilation processes relevant.

That the relic density should change so rapidly as $r$ is diminished for fixed $M_{1}$ is also not surprising when one considers the neutralino and chargino mass system. In Figure 5 the mass of the lightest and next-to-lightest neutralino are plotted as a function of the ratio $r$ when $M_{1}$ is held fixed at $M_{1}=500 \mathrm{GeV}$ and $m_{0}$ is set to $m_{0}=1000 \mathrm{GeV}$. The level crossing between a predominantly B-ino LSP to a predominantly W-ino LSP occurs abruptly at $r \simeq 0.55$. But just prior to this transition, near $r \simeq 0.65$ the degeneracy parameter $\Delta$ between the chargino and the LSP drops rapidly through the cosmologically preferred region, as is measured by the right vertical axis. Hence the justification, a posteriori, for focusing on the case $r=0.6$ in Figures 10 through 3 .

Of course, the nature of the gaugino mass system is also determined by the value of the $\mu$ parameter at the electroweak scale - and in our procedure of fixing that parameter via the EWSB conditions this implies some dependence on the gluino mass $M_{3}$ and the various scalar masses through RG running. We emphasize that the importance of the gluino mass on the relic densities is of a secondary nature, through its indirect RGE effects. Nevertheless, these effects can be significant in certain regions of parameter space.

\section{B. The case of gluino/w-ino equality}

In Section IA we looked exclusively at the case where $M_{3}=M_{1}$ at the GUT scale in order to reproduce the typical RGE behavior of universal models. In mSUGRA, with its unified gaugino masses, most of the physics we associate with a given value of $m_{1 / 2}$ is really the result of the RGE effects of the gluino. When studying neutralino relic densities the value of $M_{1}$ at the GUT scale is important in its own right, since the LSP mass is typically $m_{\chi_{1}^{0}} \simeq 0.4 M_{1}$ in such scenarios. The separate impacts of these two different mass scales are obscured when $M_{1}=M_{3}$, though this allows us to compare results to that of mSUGRA more readily.

Rather than treat the gluino mass as a completely free variable we prefer to keep the analysis tractable by invoking the relation $M_{3}=M_{2}$ in this subsection. Such a scenario will lead to gluinos which are far lighter than in the previous section, especially in the area around the critical region of $r \simeq 0.6$. What's more, we believe such a relation is likely to be closer to the truth if gaugino mass nonuniversality arises in models based on heterotic string theory.

For example, should gaugino mass nonuniversality arise at tree level in weakly-coupled string models it would probably do so via nonuniversal affine levels $k_{a}$ for the Standard Model gauge groups $\mathcal{G}_{a}$, since gaugino masses at tree level are determined by the auxiliary field of the dilaton which couples universally to the gauge groups. Standard constructions for string models enforce $k=1$ for non-abelian gauge groups such as $S U(3)$ and $S U(2)$, but generally have no such restriction on the affine factor $k_{Y}$ associated with the $U(1)$ of hy- 


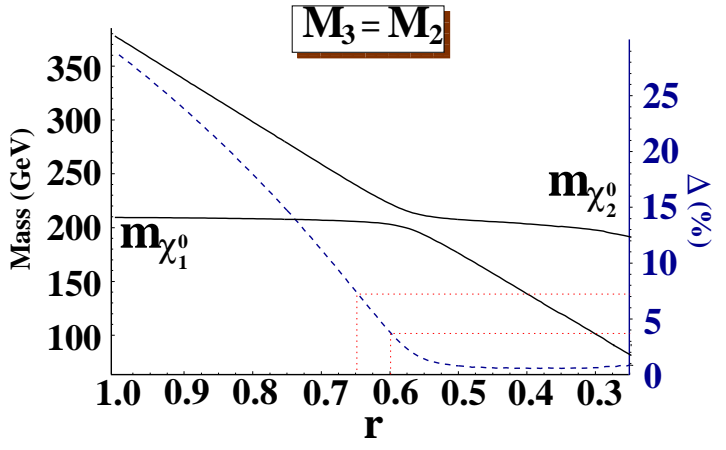

FIG. 6: Preferred Mass Degeneracy in the Gaugino System when $M_{3}=M_{2}$ and $\tan \beta=35$. The left vertical axis measures the mass (solid lines) of the lightest and nextto-lightest neutralinos $\chi_{1}^{0}$ and $\chi_{2}^{0}$, respectively, as a function of the parameter $r$. The right vertical axis measures the degree of degeneracy (dashed line) between the LSP and the chargino as given by the parameter $\Delta$, in percent, as a function of the same parameter. The cosmologically interesting region $0.65 \gtrsim r \gtrsim 0.60$ is indicated by the red dotted lines.

percharge. ${ }^{5}$ Universality at tree level is recovered when the phenomenological choice $k_{Y}=5 / 3$, corresponding to the standard GUT normalization of the hypercharge in the Standard Model, is made. But this assumption is typically not borne out in explicit string compactifications where exotic hypercharge normalizations are quite common [45, 46, 47, 48]. While such nonstandard hypercharge normalizations make gauge coupling unification less straightforward, the presence of additional states in the theory with exotic hypercharges can reconcile the Standard Model gauge couplings with the universal coupling of the underlying string theory [49].

A perhaps more palatable alternative for nonuniversalities is that the tree level gaugino masses are indeed universal, but somehow suppressed through the mechanism of supersymmetry breaking and transmission to the observable sector. Such a situation is, in fact, quite common in realistic string theories and can arise when the dilaton does not participate in supersymmetry breaking at all, or when its lowest component is stabilized using stringy nonperturbative contributions to its Kähler potential [50]. In both cases the resulting gaugino masses are loop-suppressed, implying that non-universal corrections are relevant. One class of terms arising at the oneloop level are those associated with the superconformal anomaly [51, 52] which tend to increase the gaugino mass $M_{1}$ far more than that of $M_{2}$ and $M_{3}$.

In such a regime where $M_{2} \approx M_{3}$ the level crossing that occurs in the neutralino system occurs far less abruptly. In Figure 6 we again show the masses of the LSP and

\footnotetext{
${ }^{5}$ See, for example, the discussion of this point in Ref. 44 and the references contained therein.
}

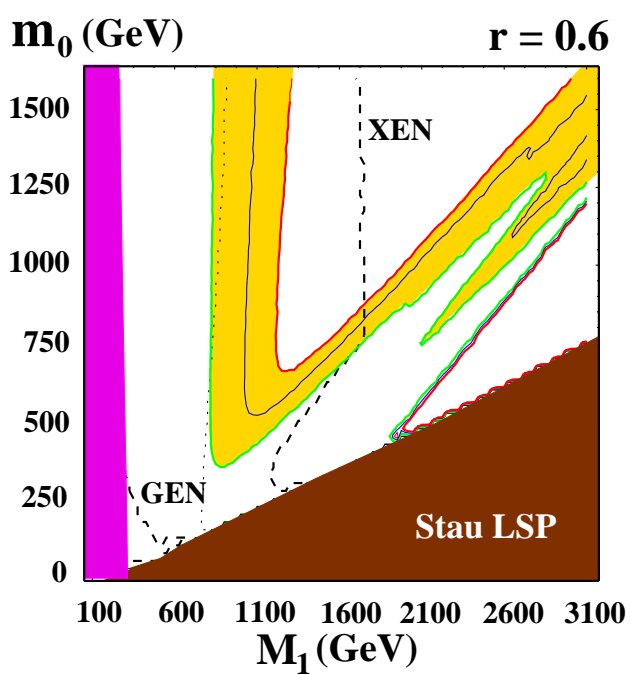

FIG. 7: Neutralino Relic Densities for $\tan \beta=5$ and $M_{3}=M_{2}$. The preferred neutralino relic density region (light shading) is displayed for $r=0.6$. The shaded regions and contours are the same as those of Figure 1 .

NLSP neutralinos for $M_{1}=500 \mathrm{GeV}, m_{0}=1000 \mathrm{GeV}$ and $\tan \beta=35$. The gluino mass at the GUT scale ranges from $500 \mathrm{GeV}$ at $r=1$ to $125 \mathrm{GeV}$ at $r=0.25$ on the right, leading to much smaller values of the resulting $\mu$ term than in Figure 5 and a smoother transition from B-ino like to W-ino like LSP. Nonetheless, the critical region where $\Delta \simeq 5 \%$ continues to fall in the area near $r=0.6$.

The effect of the lighter gluino mass is seen most dramatically in the plots analogous to those of Figure 1 . 3 . We return to the $\left(M_{1}, m_{0}\right)$ plane for $\tan \beta=5$ in Figure7, where now we have the relation $M_{3}=M_{2}=0.6 M_{1}$. As in the right panel of Figure 1 the preferred cosmological region is centered around $M_{1} \simeq 1 \mathrm{TeV}$, though this region is narrower than in the previous case. More significant is the region of rapid neutralino annihilation, and neutralino-chargino coannihilation, through heavy Higgs states that now appears even at low values of $\tan \beta$. These heavy Higgs eigenstates are lighter than in a comparable unified model because of the diminished effect of the gluino mass on the ultimate size of the $\mu$ parameter, resulting in a smaller value than in mSUGRA. In the case of Figure 7 two different resonant regions can be resolved: the lower pole region being chargino-neutralino resonant coannihilation through charged Higgs states, while the upper pole region is neutralino-neutralino resonant coannihilation through the heavy neutral Higgs states $H$ and A.

As mentioned earlier, it is well known that the size and location in the $\left(m_{1 / 2}, m_{0}, \tan \beta\right)$ space of this rapid annihilation region is a strong function of the top and bottom quark pole masses one assumes, as well as the treatment of the relevant corrections to the heavy Higgs 

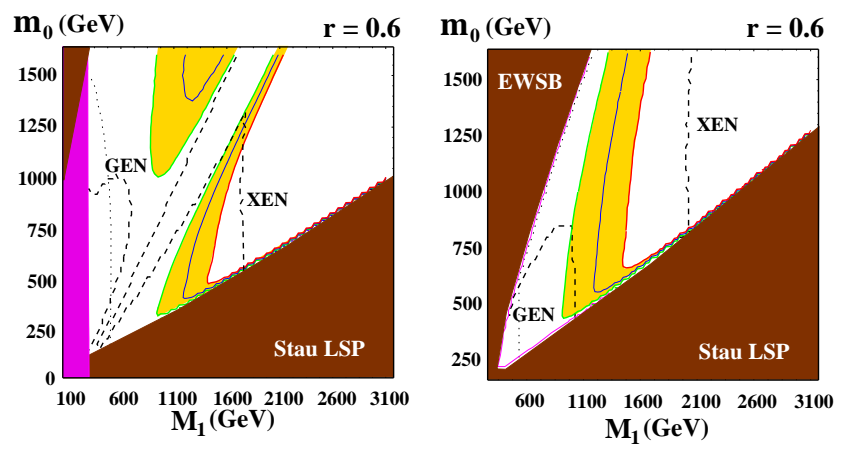

FIG. 8: Neutralino Relic Densities for $\tan \beta=35,50$ and $M_{3}=M_{2}$. The preferred neutralino relic density regions (light shading) are displayed for $r=0.6$ and $\tan \beta=35$ (left panel) and $\tan \beta=50$ (right panel). The dark shaded regions in the lower right are excluded due to a charged LSP, while those in the upper left are excluded due to improper EWSB. The medium shaded regions on the left-most edges are excluded by LEP bounds on the chargino mass. The Higgs mass contour of $m_{h}=113 \mathrm{GeV}$ is given by the dotted vertical line. The dashed contours are a rough estimate of the direct detection reach of the GENIUS ("GEN") and XENON ("XEN") experiments.

masses. ${ }^{6}$ The analysis of this section suggests that on top of this sensitivity one should add the sensitivity to the gluino mass, whose effects are felt through the RGEs.

This effect becomes even more pronounced at higher values of $\tan \beta$, as in Figure 8, where the heavier Higgs boson states become lighter still. For the case of $\tan \beta=$ 35 in Figure 8 (the left panel) the resonant annihilation region has become quite pronounced as the region where $2 m_{\chi_{1}^{0}} \approx m_{A}, m_{H}$ moves to lower values of $M_{1}$. The size of the depletion region has also increased substantially over the case where $M_{3}=M_{1}$ and $\tan \beta=50$. This is the result of resonant annihilation through the pseudoscalar Higgs being possible at lower values of $\tan \beta$, where the width $\Gamma_{A}$ is narrower and the resonance annihilation effects are more pronounced. In the center of this resonant annihilation region the neutralino relic density is very low, implying a much lower count rate for GENIUS and XENON and hence a lower direct detection reach in this area. By the time $\tan \beta=50$ is reached (the right panel in Figure 8) this resonant annihilation region no longer impacts the preferred relic density region and is confined (for this value of $r=0.6$ ) to the region $M_{1} \lesssim 1 \mathrm{TeV}$. The ultimate conclusion one should draw is that the presence of gluinos lighter than those in the universal case will gen-

${ }^{6}$ In fact, even the choices of $\alpha_{s}\left(M_{Z}\right)$ and the exact definition of the GUT scale $\Lambda_{\mathrm{UV}}=\Lambda_{\mathrm{GUT}}$ can have effects on the pseudoscalar mass large enough to effect the position of this depletion region. We have chosen the value $\alpha_{s}\left(M_{Z}\right)=0.119$ and determine the high scale $\Lambda_{\mathrm{UV}}$ by the scale at which $g_{1}$ and $g_{2}$ unify, usually close to the value of $\Lambda_{\mathrm{GUT}}=1.9 \times 10^{16} \mathrm{GeV}$.
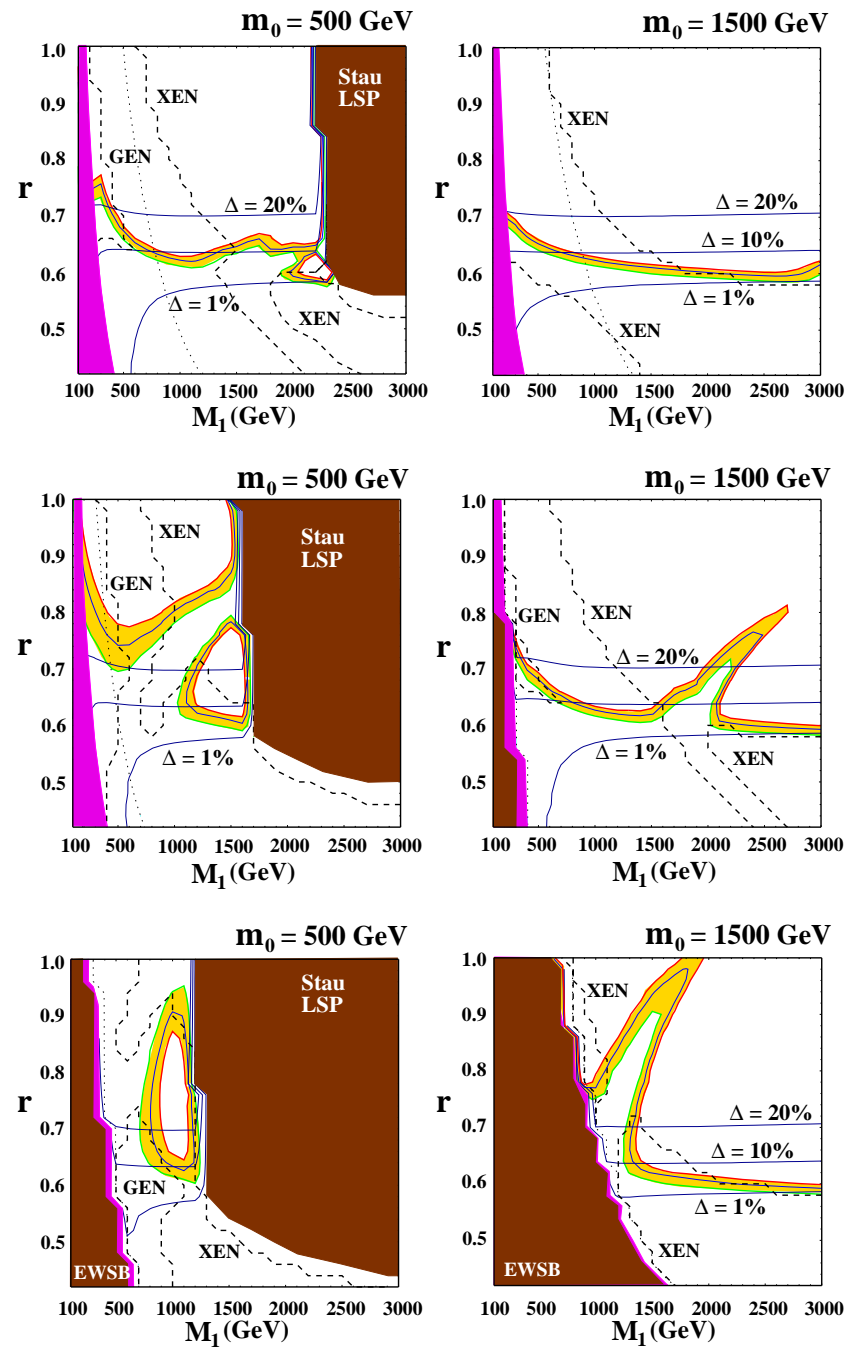

FIG. 9: Preferred Region in Gaugino Mass Space for $M_{3}=M_{2}$. Top panels are for $\tan \beta=5$, middle panels are for $\tan \beta=35$ and bottom panels are for $\tan \beta=50$. The preferred neutralino relic density regions (light shading) are displayed for universal scalar mass $m_{0}=500 \mathrm{GeV}$ (left panels) and for $m_{0}=1500 \mathrm{GeV}$ (right panels). The contours in this figure are identical to those of Figure 4.

erally imply heavy Higgs resonant annihilation at lower values of $\tan \beta$ than in mSUGRA, as well as shifting this region to higher values of the universal scalar mass for fixed $M_{1}$ and $\tan \beta$.

It should be pointed out that by choosing to look in the $\left(M_{1}, m_{0}\right)$ plane for the case where $r=0.6$ we have found that the preferred region in that plane tends to fall around $M_{1}=\mathcal{O}(1 \mathrm{TeV})$ for both choices of $M_{3}$ and all three values of $\tan \beta$. But this is an artifact of the choice $r=0.6$. The LSP mass can be arbitrarily light, up to the constraint imposed by the LEP bound on the chargino, by considering other values of $r$ for which chargino-neutralino coannihilations are less efficient. This can be seen in Figure 9, the analog to 
Figure 4 shown previously, where lighter LSP neutralinos can be obtained provided $0.7 \lesssim r \lesssim 0.9$, depending on the value of $\tan \beta$. In this region the chargino and neutralino are farther apart in mass so that $\Delta \gtrsim 20 \%$ and coannihilation processes are losing their relevance in favor of the standard annihilation processes of typical mSUGRA scenarios. This region of light charginos is also the region most accessible to GENIUS and might be favored on general fine-tuning arguments related to electroweak symmetry breaking 击. ${ }^{7}$

The distortions in Figure 9, evident for low $m_{0}$ in the $\tan \beta=5$ case and for both $m_{0}$ values in the higher $\tan \beta$ cases, are a result of the resonant annihilation region. In this region the correlation between chargino/neutralino degeneracy, as measured by $\Delta$, and the neutralino relic density is destroyed. Note also that the reduction in relic density makes this region generally inaccessible experimentally, even by the proposed XENON detector. When this distortion is factored out the detectability reach in $M_{1}$ of relic neutralinos in our local halo improves in the $M_{3}=M_{2}$ regime over the case where $M_{3}=M_{1}$.

\section{PROSPECTS FOR DIRECT DETECTION OF RELIC NEUTRALINOS}

We now wish to discuss the issue of direct detection of LSP neutralinos in more detail, couching the discussion in terms of the parameter space reach of the nextgeneration detectors such as GENIUS and XENON. To compute the spin-independent nucleon-neutralino elastic cross section the low-energy running values of the soft supersymmetry-breaking terms at the scale $M_{Z}$, as obtained by SuSpect, were passed to the Fortran code DarkSUSY [54]. The result of this analysis is the spinindependent elastic cross section $\sigma_{\chi p}^{\text {calc }}$.

The ability of a given detector to observe a signal from relic neutralino elastic scattering is a function of both the interaction cross section $\sigma_{\chi p}^{\text {calc }}$ and the number density of LSPs in the local halo. Rather than plot event rates per kilogram of target per year, we have chosen to follow the custom in theoretical work on direct detection $\mid 55]$ and plot an effective cross section as a function of the neutralino mass $m_{\chi_{1}^{0}}$. This involves a rescaling of the cross section, normalized to the case where the relic density in the cosmos is the cosmologically preferred value. In our case we have chosen the procedure outlined by Bottino et al. [55], which is to normalize the effective cross sections whenever the relic density, as computed by micrOMEGAs, is below $\left(\Omega_{\chi} \mathrm{h}^{2}\right)_{\text {calc }}=0.1$ by the ratio $\xi=\left(\Omega_{\chi} \mathrm{h}^{2}\right)_{\text {calc }} / 0.1$ :

\footnotetext{
7 These regions may also be accessible to the proposed liquidXenon based ZEPLIN 4 detector 53 whose reach is somewhat less than the GENIUS reach shown here.
}

$$
\sigma_{\chi p}^{\mathrm{eff}}=\xi \sigma_{\chi p}^{\mathrm{calc}}=\left(\frac{\left.\Omega_{\chi} \mathrm{h}^{2}\right|_{\mathrm{calc}}}{0.1}\right) \sigma_{\chi p}^{\mathrm{calc}} .
$$

This procedure allows us to consider cases where the relic density of neutralinos is far below the amount needed to provide all of the cold dark matter of the cosmos. We feel such an approach is important because (a) it represents a logical possibility given that other sources of cold dark matter can be contemplated, (b) is a more likely outcome in cases where $r<1$ than in the case of mSUGRA, and (c) points in parameter space incapable of accounting for all the needed cold dark matter are often points for which direct detection rates are relatively high. Having performed this rescaling we can now display model points together with the expected reach in cross section for various dark matter detectors. This is the manner in which the dashed detection reach contours were computed for the figures in Section 1 .

A more common method for displaying the parameter space reach of a particular detector is the manner of Figure 10. In this figure we have sampled the parameter space of Figures 1 . 3 and that of Figures 78 in intervals of $25 \mathrm{GeV}$ for $m_{0}$ and $M_{1}$. We then eliminate those points that failed to satisfy the EWSB and neutral LSP constraints, the LEP bounds on the chargino and lightest CP-even Higgs masses, or had too large a relic density $\Omega_{\chi} \mathrm{h}^{2}>0.3$. The calculated elastic cross section is then rescaled, if necessary, to produce the effective cross section $\sigma_{\chi p}^{\text {eff }}$ which is then plotted versus physical LSP mass. This procedure was performed for $\tan \beta=5$ (the top panels), $\tan \beta=35$ (middle panels) and $\tan \beta=50$ (lower panels). The left panels labeled (a) enforce $M_{3}=M_{1}$ while the right panels labeled (b) enforce $M_{3}=M_{2}$. The dark points are those of mSUGRA in which $r=1.0$ while the lighter points include both the case $r=0.60$ and $r=0.65$ to give some idea of the effect of the gaugino mass nonuniversality on the typical effective nucleon-neutralino elastic cross sections.

We should note that the reach of current and nearfuture direct detection experiments does not extend to the effective cross section range displayed in these plots. This is in accordance with the usual result for models with unified scalar masses at some large initial scale, such as mSUGRA, where the $\mu$ term tends to be large compared to gaugino masses at the electroweak scale [57, 58, 59]. Thus the LSP in all of the cases we consider has a relatively small Higgsino content. Also familiar from the unified mSUGRA case is the observation that the typical size of the elastic scattering cross section increases with higher $\tan \beta$. When the universality relation between $M_{2}$ and $M_{1}$ is relaxed (while maintaining $M_{3}=M_{1}$ ), as in the left panels of Figure 10, the increased coannihilation cross section generally depresses the effective cross section somewhat.

In the right panels of Figure 10 we tie the gluino mass to $M_{2}$. In this case the lighter gluino results in lighter squarks at the weak scale, as well as a lighter Higgs sec- 

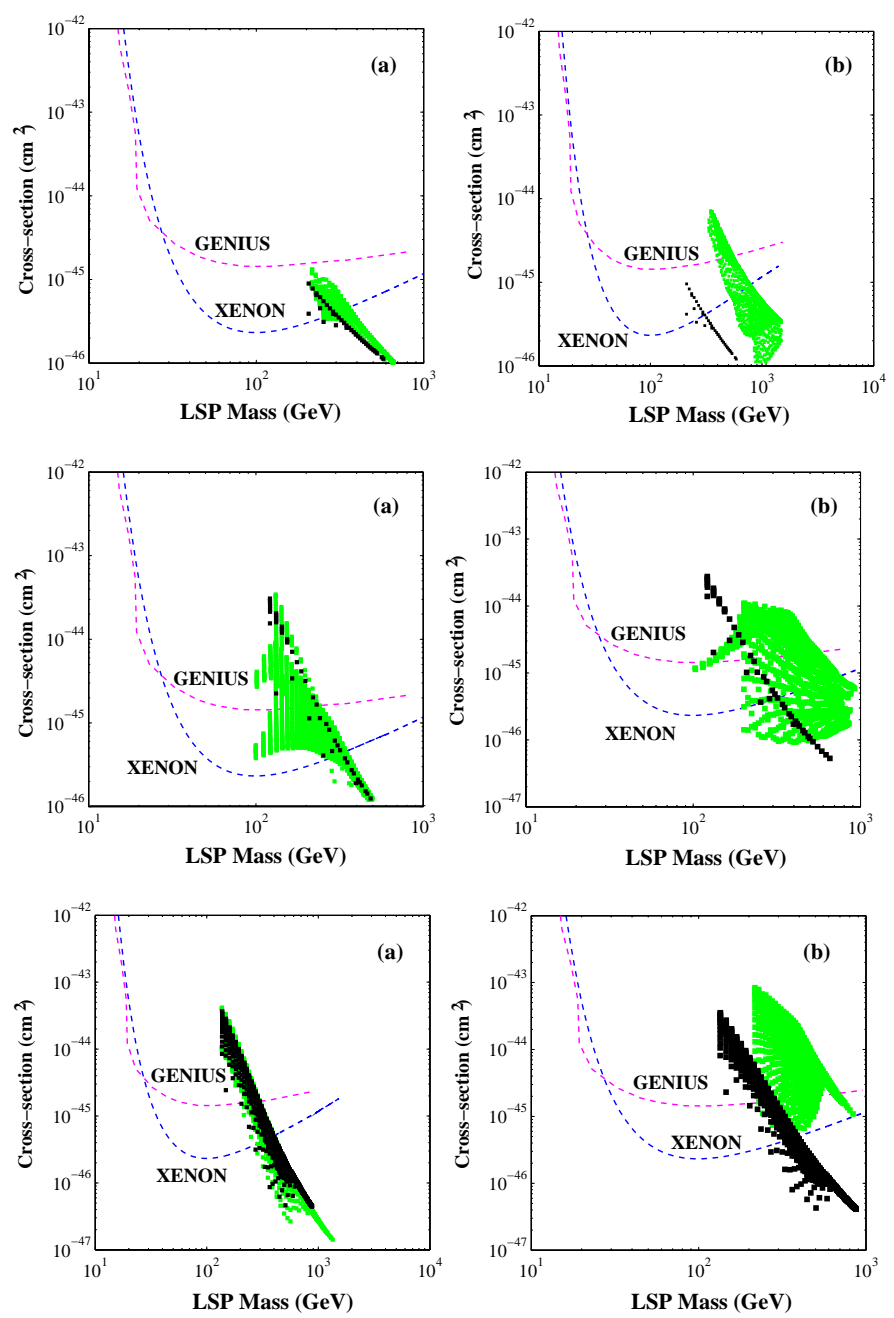

FIG. 10: Effective elastic cross sections and detector reach. The rescaled, effective spin-independent cross section $\sigma_{p \chi}^{\text {eff }}$ is plotted versus the physical LSP mass $m_{\chi_{1}^{0}}$ for $\tan \beta=5$ (top panels), $\tan \beta=35$ (middle panels) and $\tan \beta=50$ (bottom panels). The left panels labeled (a) enforce the relation $M_{3}=M_{1}$ of Section I A while the right panels labeled (b) enforce the relation $M_{3}=M_{2}$ of Section IB. Dark (black) points are those of mSUGRA where $r=1$. The lighter (green) points are those in the region $0.60 \leq r \leq 0.65$. The estimation of the GENIUS and XENON detector reaches was obtained via the dark matter plotting program of Ref. 56 .

tor though the smaller value of $\mu$ that results. This dramatically increases the importance of those squark and Higgs exchange diagrams that contribute to the spinindependent elastic cross section for neutralinos on nucleons. The cross section in this paradigm is augmented by one to two orders of magnitude: in particular we note that at large $\tan \beta$ nearly the entire range of parameter space shown will be covered by the proposed XENON experiment. This marked increase in the elastic cross section is consistent with a wide array of previous studies. The reason is clear: any physics which reduces the size of the low-energy squark and Higgs masses - whether directly through modifying the soft terms or indirectly through RG effects - will increase the importance of those squark and Higgs exchange diagrams that contribute to the spin-independent elastic cross section for neutralinos on nucleons. This includes the gaugino mass nonuniversalities that lower $M_{3}$ [9], beginning the RG evolution from a lower energy scale [60], or studying models with non-universal scalar masses 61, 62.

\section{FINE-TUNING AND SENSITIVITY ANALYSIS}

The question of fine-tuning in a given parameter, or set of parameters, comes down to asking how natural it is to end up with the parameter at a certain value. Such a determination should ultimately be done in the context of some underlying theory, such as string theory. Without the luxury of such a fundamental theory we are forced to rely on constructed notions of fine-tuning. The area of fine-tuning has attracted many different definitions and interpretations. ${ }^{8}$ We are not especially fond of any particular measure of tuning. Nonetheless, we think that a firm, standard definition should be used when comparing models and regions of parameter space. There are two common measures of fine-tuning employed when discussing relic densities.

First is a frequent, though soft, definition of fine-tuning we might call the "optical" or "likelihood" method. Here one simply looks at a plot of parameter space and "eyeballs" whether or not the allowed parameter space is finetuned by estimating the relative sizes of the preferred volume of parameter space to the entire volume otherwise allowed in the theory. For instance, looking at the $\tan \beta=5$ plot in Figure 1 for mSUGRA (the $r=1$ limit for the rSUGRA models), one notices that the relic density depends on a very specific ratio of $m_{0}$ to $M_{1 / 2}=M_{1}$. Since only a very thin sliver of parameter space meets the relic density constraint, one would conclude, using the likelihood method, that dark matter is fine-tuned in $\operatorname{mSUGRA}$ for $\tan \beta=5$. As we will see below, this is not necessarily the case when more robust measures of fine-tuning are employed.

The optical method can be used on the rSUGRA parameter space to come to any desired conclusion about fine-tuning. For instance, looking at the $M_{1}$ vs. $m_{0}$ plot for $r=0.6$ in Figure 1, the preferred dark matter region does not look especially tuned: a wide region exists that meets the relic density constraint. However, the opposite conclusion is reached if one looks instead at the $M_{1}$ vs. $r$ plot for $\tan \beta=5$ in Figure 4 . As noted earlier, on this plot the parameter space looks quite narrow and is obviously rather dependent on $r$.

To eliminate this ambiguity, in this paper we choose

${ }^{8}$ See, for example, the discussion of this point in Ref. 63 . 
the second and slightly less subjective definition commonly employed, which is based on a "sensitivity" measure 64, 65] as applied to neutralino relic densities by Ellis and Olive [5]

$$
\Delta_{\Omega, i} \equiv \frac{a_{i}}{\Omega_{\chi} \mathrm{h}^{2}} \frac{\partial\left(\Omega_{\chi} \mathrm{h}^{2}\right)}{\partial a_{i}}
$$

The $a_{i}$ in (7) represent parameters that are to be varied in the theory, typically soft breaking parameters though sometimes also the top and bottom Yukawa couplings or masses. Even though this definition is not without limitations [66], we find it at least provides a common language which makes comparisons easier. The overall level of fine-tuning then is given by

$$
\Delta_{\Omega}=\sqrt{\sum_{i}\left(\Delta_{\Omega, i}\right)^{2}} .
$$

We will compute two different $\Delta_{\Omega}$ 's for certain sample points in the generalized rSUGRA parameter space. In the first we compute $\Delta_{\Omega}$ for the case where the $a_{i}$ belong to the set $\left\{r, m_{0}, M_{1}, \tan \beta, m_{t}, m_{b}\right\}$. Since we will restrict ourselves to one of the gluino mass relations of Sections $\overline{I A}$ and $[\mathrm{IB}$, this parameter set represents the maximal sensitivity measure for a given point. But we will also consider the case where a more fundamental theory predicts one specific value of $r$, so we will also compute the quantity $\Delta_{\Omega}^{y}$, where the $a_{i}$ now belong to the smaller set $\left\{m_{0}, M_{1}, \tan \beta, m_{t}, m_{b}\right\}$. For example, the variation of $r$ in mSUGRA has no meaning, since $r=1$ is one of the defining characteristics of this model. ${ }^{9}$ Thus we will only compute the quantity $\Delta_{\Omega}^{y}$ for the mSUGRA scenario as a baseline for comparison with the expanded parameter space of rSUGRA.

We begin our sensitivity analysis by considering the

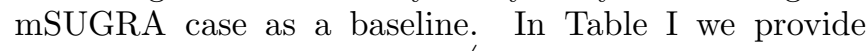
the sensitivity parameter $\Delta_{\Omega}^{\gamma}$ for a sample of points which yield the cosmologically preferred relic density (the shaded areas in the left panels of Figures 1, 2 and 3). These values are in very good agreement with those calculated in Ref. 5 for similar points in the parameter space. The sensitivity is greatest for large $\tan \beta$, which is expected due to the presence of the resonant annihilation area which is itself sensitive to $\tan \beta$ and $m_{b}^{\text {pole }}$.

Let us compare these typical values with those of various points in the rSUGRA parameter space. Table [1] looks at the sensitivity parameters for a set of points under three different gaugino mass relations: $M_{3}=M_{1}$, $M_{3}=M_{2}$, and $M_{2}=M_{1}$. The first two represent the case of Sections $[\mathrm{IA}$ and $\mathrm{IB}$, respectively, while the third

\footnotetext{
${ }^{9}$ Of course the most well-studied unified model to lay claim to a string theory origin - namely that of the dilaton domination model at tree-level - predicts even more constraints among the various soft parameters of the mSUGRA paradigm.
}

TABLE I: Relic Density Sensitivity for Preferred Points in mSUGRA Parameter Space.

\begin{tabular}{|ccc||c|}
\hline$M_{1}$ & $m_{0}$ & $\tan \beta$ & $\Delta_{\Omega}^{\eta}$ \\
\hline 1100 & 235 & 5 & 13.0 \\
1500 & 343 & 5 & 15.7 \\
1100 & 390 & 35 & 31.8 \\
1100 & 550 & 50 & 78.3 \\
1100 & 950 & 50 & 124.1 \\
1600 & 740 & 50 & 44.2 \\
1600 & 1500 & 50 & 131.9 \\
\hline
\end{tabular}

TABLE II: Relic Density Sensitivity for Preferred Points in rSUGRA Parameter Space.

\begin{tabular}{||cccc||cc||cc||cc||}
\multicolumn{1}{c|}{ Point } & \multicolumn{1}{c|}{$M_{3}=M_{1}$} & $M_{3}=M_{2}$ & \multicolumn{2}{c}{$M_{2}=M_{1}$} \\
\hline$M_{1}$ & $m_{0}$ & $\tan \beta$ & $r$ & $\Delta_{\Omega}$ & $\Delta_{\Omega}^{\eta}$ & $\Delta_{\Omega}$ & $\Delta_{\Omega}^{\eta}$ & $\Delta_{\Omega}$ & $\Delta_{\Omega}^{\eta}$ \\
\hline 900 & 350 & 5 & 0.60 & 50.6 & 3.3 & 39.2 & 15.4 & 6.6 & 4.5 \\
1100 & 1500 & 5 & 0.60 & 54.0 & 3.4 & 50.1 & 7.7 & 5.4 & 5.2 \\
3000 & 1500 & 5 & 0.60 & 48.9 & 2.5 & 40.7 & 16.3 & 250.2 & 218.6 \\
3000 & 1375 & 5 & 0.60 & 48.8 & 2.5 & 49.4 & 39.4 & 53.6 & 47.5 \\
3000 & 1250 & 5 & 0.60 & 48.7 & 2.5 & 86.1 & 73.5 & 56.2 & 49.7 \\
1100 & 1500 & 35 & 0.60 & 53.5 & 3.3 & 47.6 & 17.2 & 70.7 & 69.8 \\
1100 & 1000 & 35 & 0.60 & 50.0 & 2.5 & 73.6 & 68.9 & 70.9 & 68.8 \\
1600 & 1500 & 35 & 0.60 & 51.5 & 2.6 & 80.7 & 74.4 & 115.5 & 112.7 \\
2100 & 1500 & 35 & 0.60 & 47.8 & 2.7 & 24.0 & 6.0 & 58.2 & 57.8 \\
400 & 500 & 35 & 0.67 & 70.8 & 62.9 & 22.5 & 22.3 & 136.1 & 136.0 \\
300 & 1500 & 35 & 0.75 & 9.9 & 9.9 & 20.3 & 18.9 & 18.0 & 17.4 \\
1100 & 750 & 50 & 0.60 & 27.8 & 23.1 & 26.8 & 6.0 & 8.4 & 7.8 \\
1600 & 1500 & 50 & 0.60 & 39.8 & 32.7 & 28.6 & 7.2 & 19.7 & 18.8 \\
\hline
\end{tabular}

enforces $M_{3}=r M_{1}=r M_{2}$. This third relation was studied by the authors of Ref. 9 and we include it here to make contact with their observations. For these rSUGRA scenarios we have chosen points which have the correct relic density in the $M_{3}=M_{2}$ case (Figure 9), but many of the points also have the correct relic density in the $M_{3}=M_{1}$ case so the comparison is still instructive.

One can see that when $r$ represents the ratio of $M_{2}$ to $M_{1}$, as it does in the first two columns of Table [1, its inclusion in the set $\left\{a_{i}\right\}$ has an important effect on the overall degree of sensitivity. This is especially striking in the first column in cases where the LSP is quite massive (large $M_{1}$ ) where the correct relic density is obtained almost exclusively through coannihilation effects. Here the sensitivities are everywhere comparable to those of mSUGRA in Table If. The large impact of including $r$ in the set $\left\{a_{i}\right\}$ is merely verifying that this one parameter effectively captures the key physics involved in the relic density calculation.

In the second and third column, where the gluino mass is allowed to vary and is generally smaller than in the first column, the impact of the variable $r$ is somewhat muted. As mentioned previously, the impact of $M_{3}$ is on the lower squark masses and the lower value of $\mu$. In the extreme case of the third column of Table II, where $M_{2}=M_{1}$ and 
only the gluino mass is allowed to vary (downwards) we see that the impact of the relative size between $M_{3}$ and the other gaugino masses is minor. What is far more important is the absolute magnitude of $M_{3}$, particularly in points associated with the region near a Higgs resonance. In these examples the fine tuning is comparable to that of mSUGRA near a Higgs resonance and is $\mathcal{O}(100)$ in size. This indicates that when resonant annihilation processes are important it is the gluino mass at the high scale that captures the largest fraction of the physics involved.

Thus, in the worst case scenario for $M_{2}$-floating rSUGRA, the relic density sensitivity is a factor of 4 larger than a comparable point in mSUGRA parameter space. In the best case scenario, rSUGRA relic density sensitivities are better than comparable fine-tunings in mSUGRA parameter space by a factor of 4 . This is true even ignoring the possibility that $r$ might not be included in the set of variation parameters. If $r$ is excluded, the relic density fine-tunings of rSUGRA compare even more favorably with those of mSUGRA.

So what are we to conclude from Tables II and II] On the one hand we agree with the authors of Ref. 9: achieving the preferred cosmological relic density requires that the parameter $r$ be tuned to a specific narrow range for any given universal scalar mass $m_{0}$, B-ino mass $M_{1}$ and $\tan \beta$. But the same could be said for any model that predicts a stable relic neutralino. In mSUGRA one must tune $\tan \beta$ and/or the mass ratio of the stau and the lightest neutralino to a specific narrow range to get the right answer. In cases where the gluino is separated from the (still unified) B-ino and $\mathrm{W}$-ino masses one must tune its value to a narrow range in order to achieve just the right value of $\mu$ at the electroweak scale. Tuning of this type is common to phenomenology where we lack a fundamental theory that can explain such relations.

On the other hand we also agree with the authors of Ref. 5: these tunings that are required of the theory are generally speaking not fine-tunings. That is to say, the sensitivity to input parameters represented by the values for $\Delta_{\Omega}$ in Tables II and II are on the whole quite mild. In fact the relatively small tuning parameters $\Delta_{\Omega}$ are all the more surprising when we recall that calculating the relic density in the presence of coannihilations involves the exponential of the mass difference $\Delta$ of (5). If a stable neutralino is a significant component of the cold dark matter of the cosmos (and we reiterate that it certainly need not be), then some specific area of the MSSM parameter space will inevitably be singled out. But it is worth bearing in mind that the tuning of parameters implied in Tables If and II is generally small in comparison with the tuning required in the electroweak symmetrybreaking sector to obtain the correct $Z$-boson mass - a physically measured quantity.

\section{Conclusion}

Rapidly advancing calculational tools have put the computation of many important observables relating to relic neutralinos at hand to an ever-widening community. We believe making sound judgements as to the region of supersymmetric model space favored by cosmological observations on the density of cold dark matter requires utilizing these tools in directions beyond that of unified models. In this work we have focused on the case of nonuniversal gaugino masses and, in particular, a paradigm in which the $\mathrm{W}$-ino mass $M_{2}$ (and perhaps the gluino mass) are significantly smaller at the high-energy input scale than the B-ino mass $M_{1}$. Such a regime may be a likely outcome of effective supergravity theories based on the weakly-coupled heterotic string, though we have presented the implications of these nonuniversalities in a model-independent manner.

Many complications and uncertainties exist that should give us pause in trying to make exact quantitative statements: broad theoretical questions such as whether there may be non-thermal LSP production mechanisms or whether phase transitions at late times may have generated some degree of late-term inflation. There are also technical issues that can have a significant impact on one's results, particularly at large $\tan \beta$ where the choice of physical pole masses for the quarks, the accuracy of the RG evolution employed, and the completeness of the corrections to Higgs masses and widths are all crucial.

Nonetheless, it is clear that relaxing the unification condition on gaugino masses opens up new directions in the parameter space which are every bit as theorymotivated and phenomenologically appealing as those of mSUGRA. These new directions may improve the prospects for detecting relic neutralinos from our halo in future experiments, particularly the proposed liquid Xenon experiments. The necessary tunings involved may, in fact, be a tool in helping us to ascertain the correct underlying theory once a discovery is made (for example, selecting a narrow list of possible hidden sector condensing gauge groups in string-derived supergravity models [3]). In this sense the work presented here on nonuniversal gaugino masses forms an attractive complement to the recent work on nonuniversal Higgs masses [24, 67. Both are well-defined departures from the baseline mSUGRA paradigm which can claim solid motivation from heterotic string inspired models. Computation of the relic density and elastic scattering cross section for relic neutralino LSPs is a wonderful laboratory for studying supersymmetric phenomenology in that it combines elements from the squark, slepton, gaugino and Higgs sectors in interrelated ways. We believe disentangling these diverse physics contributions is best performed with a toolkit that contains a variety of more general MSSM models with which to work. 


\section{Acknowledgments}

The authors would like to thank M. K. Gaillard for helpful conversations and suggestions. BDN would like to thank the Lawrence Berkeley National Laboratory for hospitality during the early portions of this work.
[1] N. Bachall, J. P. Ostriker, S. Perlmutter and P. J. Steinhardt, Science 284 (1999) 1481.

[2] W. L. Freedman, Phys. Rept. 333-334 (2000) 1.

[3] A. Birkedal-Hansen and B. D. Nelson, Phys. Rev. D64 (2001) 015008.

[4] G. L. Kane, J. Lykken, B. D. Nelson and L. Wang, Reexamination of Electroweak Symmetry Breaking in Supersymmetry and Implications for Light Superpartners, hep-ph/0207168.

[5] J. Ellis and K. A. Olive, Phys. Lett. B514 (2001) 114.

[6] M. Brhlik, D. J. H. Chung and G. L. Kane, Int. J. Mod. Phys. D10 (2001) 367.

[7] S. Mizuta, D. Ng and M. Yamaguchi, Phys. Lett. B300 (1993) 96.

[8] A. Corsetti and P. Nath, Phys. Rev. D64 (2001) 125010.

[9] V. Bertin, E. Nezri and J. Orloff, Neutralino Dark Matter Beyond CMSSM Universality, hep-ph/0210034.

[10] A. Djouadi, J. L. Kneur and G. Moultaka, SuSpect: A Program for the Supersymmetric Spectrum, hep$\mathrm{ph} / 9901246$,

[11] G. Belanger, F. Boudjema, A. Pukhov and A. Semenov, MicrOMEGAs: A Proqram for Calculating the Relic Density in the MSSM, hep-ph/0112278, http://wwwlapp.in2p3.fr/lapth/micromegas.

[12] K. A. Olive and M. Srednicki, Nucl. Phys. B355 (1991) 208.

[13] P. Gondolo and G. Gelmini, Nucl. Phys. B360 (1991) 145 .

[14] M. Drees and M. M. Nojiri, Phys. Rev. D47 (1993) 376.

[15] G. Jungman, M. Kamionkowski and K. Griest, Phys. Rept. 267 (1996) 195.

[16] V. Barger and C. Kao, Phys. Rev. D57 (1998) 3131.

[17] K. Griest and D. Seckel, Phys. Rev. D43 (1991) 3191.

[18] J. Edsjo and P. Gondolo, Phys. Rev. D56 (1997) 1879.

[19] J. Ellis, T. Falk, K. A. Olive and M. Srednicki, Astropart. Phys. 13 (2000) 181.

[20] J. Ellis, K. A. Olive and Y. Santoso, Calculations of Neutralino-Stop Coannihilation in the CMSSM, hep$\mathrm{ph} / 0112113$.

[21] H. Baer, C. Balázs and A. Belyaev, JHEP 0203 (2002) 042 .

[22] T. Nihei, L. Roszkowski and R. R. de Austri, JHEP 0207 (2002) 024.

[23] A. Birkedal-Hansen and E. Jeong, Gaugino and Higgsino Coannihilations I: Neutralino-Neutralino Interactions, hep-ph/0210041.

[24] J. Ellis, T. Falk, K. A. Olive and Y. Santoso, Exploration of the MSSM with Non-Universal Higgs Masses, hep-ph/0210205.

[25] J. Ellis, T. Falk, G. Ganis and K. A. Olive, Phys. Rev. D62 (2000) 075010.

[26] J. L. Feng, K.T. Matchev and F. Wilczek, Phys. Lett. B482 (2000) 388.

[27] V. Barger and C. Kao, Phys. Lett. B518 (2001) 117.

[28] A. Djouadi, M. Drees and J. L. Kneur, JHEP 0108
(2001) 055 .

[29] H. Baer, C. Balázs, A. Belyaev, J. Mizukoshi, X. Tata and Y. Wang, JHEP 0207 (2002) 050.

[30] A. Melchiorri and J. Silk, Phys. Rev. D66 (2002) 041301.

[31] CLEO Collaboration, $B$ to $s$ gamma Branching Fraction and CP Asymmetry, CLEO CONF 99-10, hepex/9908022.

[32] S. P. Martin and J. D. Wells, Super-conservative Interpretation of Muon g-2 Results Applied to Supersymmetry, hep-ph/0209309.

[33] Muon g-2 Collaboration, Phys. Rev. Lett. 89 (2002) 101804.

ERRATUM Phys. Rev. Lett. 89 (2002) 129903.

[34] ALEPH Collaboration, Phys. Lett. B499 (2001) 67.

[35] T. Alderweireld, MSSM SUGRA Searches at LEP, Talk given at XXXVI Rencontres de Moriond, Les Arcs, France, March 17-24 2001, hep-ex/0107083.

[36] ALEPH Collaboration, Phys. Lett. B526 (2002) 191.

[37] L. Baudis et al., Phys. Rept. 307 (1998) 301.

[38] E. Aprile et al., XENON: A 1 Ton Liquid Xenon Experiment for a Sensitive Dark Matter Search, astro$\mathrm{ph} / 0207670$.

[39] J. Ellis, D. V. Nanopoulos and K. A. Olive, Phys. Lett. B508 (2001) 65.

[40] J. Ellis, T. Falk, G. Ganis, K. A. Olive and M. Srednicki, Phys. Lett. B510 (2001) 236.

[41] T. Nihei, L. Roszkowski and R. R. de Austri, JHEP 0203 (2002) 031.

[42] A. B. Lahanas and V. C. Spanos, Eur. Phys. J. C23 (2002) 185.

[43] T. Moroi and L. Randall, Nucl. Phys. B570 (2000) 455.

[44] K. R. Dienes, Phys. Rept. 287 (1997) 447.

[45] K. R. Dienes, A. E. Faraggi, J. March-Russell, Nucl. Phys. B467 (1996) 44.

[46] S. Chaudhuri, G. Hockney and J. D. Lykken, Nucl. Phys. B469 (1996) 357.

[47] G. B. Cleaver, A. E. Faraggi and C. Savage, Phys. Rev. D63 (2001) 066001.

[48] J. Giedt, Annals Phys. 297 (2002) 67.

[49] J. Giedt, Optical Unification, hep-ph/0205224.

[50] G. L. Kane, J. Lykken, S. Mrenna, B. D. Nelson, L. Wang and T. T. Wang, Theory-Motivated Benchmark Models and Superpartners at the Tevatron, hep-ph/0209061.

[51] M. K. Gaillard, B. D. Nelson and Y.-Y. Wu, Phys. Lett. B459 (1999) 549.

[52] J. Bagger, T. Moroi and E. Poppitz, JHEP 0004 (2000) 009.

[53] D. B. Cline, Nucl. Phys. Proc. Suppl. 87 (2000) 114.

[54] P. Gondolo, J. Edsjo, L. Bergstrom, P. Ullio and E. A. Baltz, DarkSUSY - A Numerical Packaqe for Dark Matter Calculations in the MSSM, astro-ph/0012234,

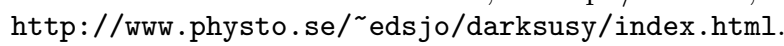

[55] A. Bottino, N. Fornengo and S. Scopel, Nucl. Phys. B608 (2001) 461.

[56] R. Gaitskill and V. Mandic, 
http://dmtools.berkeley.edu/limitplots/

[57] A. B. Lahanas, D. V. Nanopoulos and V. C. Spanos, Phys. Lett. B518 (2001) 94.

[58] J. Ellis, A. Ferstl and K. A. Olive, Phys. Lett. B532 (2002) 318.

[59] Y. G. Kim, T. Nihei, L. Roszkowski and R. R. de Austri, Upper and Lower Limits on Neutralino WIMP Mass and Spin-Independent Cross Section, hep-ph/0208069.

[60] E. Gabrielli, S. Khalil, C. Muñoz and E. Torrente-Lujan, Phys. Rev. D63 (2001) 025008.

[61] R. Arnowitt, B. Dutta, B. Hu and Y. Santoso, Phys. Lett. B505 (2001) 177.
[62] Y. G. Kim and M. M. Nojiri, Prog. Theor. Phys. 106 (2001) 561.

[63] J. L. Feng and K. T. Matchev, Phys. Rev. D63 (2001) 095003.

[64] J. Ellis, K. Enqvist, D. Nanopoulos and F. Zwirner, Nucl. Phys. B276 (1986) 14.

[65] R. Barbieri and G. Giudice, Nucl. Phys. B306 (1988) 63.

[66] G. Anderson and D. Castaño, Phys. Lett. B347 (1995) 300 .

[67] J. Ellis, K. A. Olive and Y. Santoso, Phys. Lett. B539 (2002) 107. 